\title{
Functors for Proofs and Programs
}

\author{
Jean-Christophe Filliâtre and Pierre Letouzey \\ LRI - CNRS UMR 8623 \\ Université Paris-Sud, France \\ $\{$ filliatr,letouzey\}@lri.fr
}

\begin{abstract}
This paper presents the formal verification with the Coq proof assistant of several applicative data structures implementing finite sets. These implementations are parameterized by an ordered type for the elements, using functors from the ML module system. The verification follows closely this scheme, using the newly Coq module system. One of the verified implementation is the actual code for sets and maps from the Objective Caml standard library. The formalization refines the informal specifications of these libraries into formal ones. The process of verification exhibited two small errors in the balancing scheme, which have been fixed and then verified. Beyond these verification results, this article illustrates the use and benefits of modules and functors in a logical framework.
\end{abstract}

\section{Introduction}

Balanced trees are notoriously hard to implement without mistakes. Exact invariants are difficult to figure out, even for applicative implementations. Since most programming languages provide data structures for finite sets and dictionaries based on balanced trees, it is a real challenge for formal verification to actually verify one of these.

We choose to verify the set module from the Objective Caml (OCAML) standard library 2. This applicative implementation of finite sets uses AVL trees 4] and provides all expected operations, including union, difference and intersection. Above all, this is a very efficient and heavily used implementation, which motivates a correctness proof. In particular, a formalization requires to give precise specifications to this library, a non-trivial task. This article also presents the verification of two other implementations, using respectively sorted lists and red-black trees [8], both written in OCAML and using the same interface as Set.

Building balanced trees over values of a given type requires this type to be equipped with a total ordering function. Several techniques are available to build a parametric library: in ML, polymorphism gives genericity over the type and first-class functions give the genericity over the ordering function (e.g. it is passed when initially creating the tree); in object oriented languages, objects to be stored in the trees are given a suitable comparison function; etc. The most elegant technique is probably the one provided by the ML module system, as implemented in SML [9] and OCAML [11]. A module is a collection of definitions 
of types, values and submodules. Its type is called a signature and contains declarations of (some of the) types, values and submodules. Modules can be parameterized by some signatures and later applied to actual modules. Such functions from modules to modules are called functors. The Set library is a nice illustration of the OCAML module system. It is naturally written as a functor taking the signature of an ordered type as argument and returning a signature for finite sets as a result.

The use of modules and functors introduces an extra challenge for formal proof. The recent introduction of an ML-like module system into the CoQ proof assistant 15] makes it a perfect candidate for this verification. As a side effect, this article examplifies the use of modules and functors in a logical framework, which goes well beyond its use in programming languages.

Currently, CoQ is not able to reason directly about OcAmL code. To certify an OCAML applicative implementation, we first translate it into CoQ's own programming language, GallinA. Then the logic of CoQ can be used to express properties of the GALLinA functions. Whereas the translation from OCAML to Gallina is done manually and is thus error-prone, CoQ provides an automated mechanism for the converse translation called extraction. The extraction takes a COQ function or proof, removes all its logical statements, and translates the remaining algorithmic content to OCAML. We end up with three versions of the code: the OCAML original handwritten one, its GALLINA translation certified in COQ, and the OCAML extracted version. Theoretical results about the extraction $[12,13]$ ensure that the extracted code verifies the same properties as the GALlinA version.

Even if in this case the extracted code is not aimed at replacing the existing one, there are at least two reasons to perform this extraction. First, the extracted code often presents only syntactical differences with respect to the original code and thus ensures that no mistake was made during the hand-translation to CoQ. Second, the extracted code behaves reasonably well in practice (see e.g. the final benchmarks) and thus shows that the extraction technique could be used directly without any original OCAML code.

Outline. This paper is organized as follows. Section 2 is devoted to the presentation of OCAML and COQ module systems. Section 3 introduces the signatures for ordered types and finite sets, and various utility functors over these signatures. Section 4 presents the verification of three finite sets implementations, using respectively sorted lists, AVL trees from the OCAML standard library and red-black trees. Section 5 concludes with a benchmark comparing performances of handwritten and extracted code.

Source code. This article only details the most important parts of the formal development. The whole source code would take too much space, even in appendix: the sole specification is 2472 lines long and the proof scripts amount to 5517 lines, not mentioning the original source code for AVL and red-black trees. All these files are available at http://www.lri.fr/ filliatr/fsets/ for downloading and browsing. The COQ files need the development version of CoQ to compile and a rather powerful machine: 10 minutes are indeed necessary to compile the 
whole development on a 1 Gigahertz Intel CPU. This web site also includes a slightly more detailed version of this paper.

The pieces of COQ and OCAML code given in this article are displayed in verbatim font, apart from the embellishment of a few symbols: $\rightarrow$ for $\rightarrow, \leftrightarrow$ for $<->, \vee$ for $\backslash, \wedge$ for $\wedge, \neg$ for $\sim, \forall$ for universal quantifiers and $\exists$ for EX.

\section{Modules and Functors}

\subsection{The OCAmL Module System}

The OCAML module system [1] is derived from the original one for SML 9 . The latter also evolved in return, both systems being now quite close and known as the Harper-Lillibridge-Leroy module system. This section briefly illustrates the OCAML module system with the Set library from its standard library, which signature is used throughout this paper and which code is verified in Section 4.2

The Set library first defines a signature $\mathbf{S}$ for finite sets, given Figure 1 It contains the type elt of elements, the type $t$ of sets, the value empty for the empty set, and 22 operations over sets. Most of them have an obvious meaning and the expected semantics. Other like fold, elements or choose have part of their specification left unspecified (this is detailed later in this paper).

The set implementation is parameterized by the type of its elements, which must be equipped with a total ordered function. The signature OrderedType is introduced for this purpose:

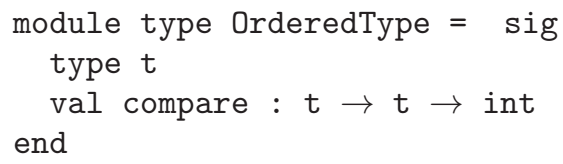

The compare function is returning an integer such that (compare $\mathrm{x} y$ ) is zero if $\mathrm{x}$ and $\mathrm{y}$ are equal, negative if $\mathrm{x}$ is smaller than $\mathrm{y}$ and positive if $\mathrm{x}$ is greater than y. For instance, a module Int realizing this signature for the type int of integers and the predefined comparison function Pervasives.compar 1 would be:

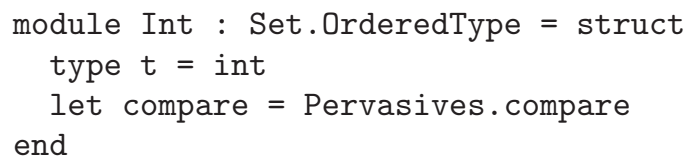

The implementation of the data structure for sets is provided as a functor Make taking a module Ord of signature OrderedType as argument and returning a module of signature $\mathrm{S}$ :

module Make (Ord : OrderedType) : S with type elt = Ord.t

${ }^{1}$ The subtraction can not be used as comparison function for integers because of overflows; consider for instance min_int $-\max _{-}$int $=1$. 


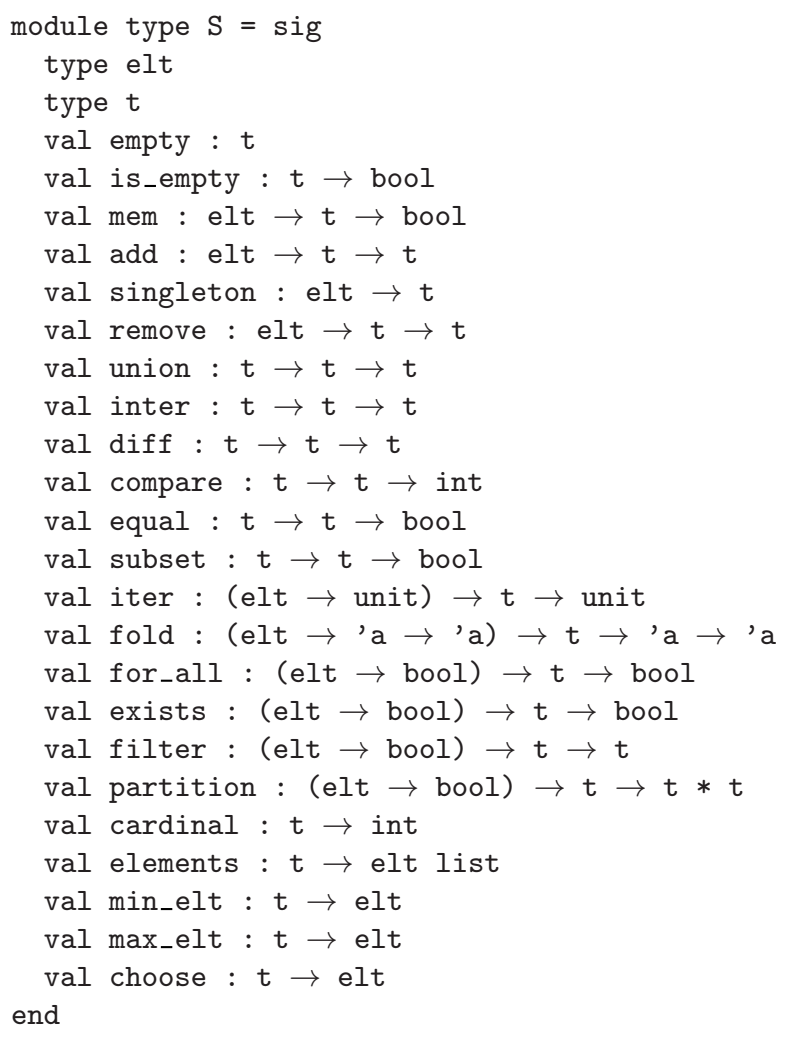

Fig. 1. OCAML signature Set.S for finite sets

The signature for the returned module needs to be more precise than S: we must identify the type elt in the returned signature with the type Ord.t given as argument. This is made explicit using the with type construct.

We would get sets of integers by applying this functor to the module Int above: module IntSet $=$ Set. Make (Int). It is important to notice that signature $\mathrm{S}$ contains a type $\mathrm{t}$ and a comparison function compare (with the expected behavior), allowing to build sets of sets by applying Make again on the resulting module. For instance, sets of sets of integers would be obtained with: module IntSetSet $=$ Set. Make $($ IntSet $)$. The reader may refer to the OCAML manual [2] for further details on its module system.

\subsection{The Coq Module System}

A module system for the CoQ proof assistant has been advocated for a long time, mainly in Courant's PhD thesis [7. Clearly more ambitious than OCAML's, his module system appeared too complex to be implemented in the existing code 
of Coq. Instead, a module system à la Caml was recently implemented by Chrząszcz [5] following Leroy's modular module system [11].

One small difference with respect to OCAML is that COQ modules are interactive. While in OCAML a module is introduced as a single declaration, COQ allows to build it piece by piece. The declaration of a new module M starts with the command Module M. Then any subsequent COQ declaration (type, function, lemma, theorem, etc.) is placed in that module, until the closing of $\mathrm{M}$ with the command End M. This interactive process is plainly justified by the interactive building of (most) COQ theories. Developing a whole module with all its proofs one at a time would be intractable. Signatures and functors are also introduced interactively in a similar way.

A signature may contain a declaration such as Parameter $\mathrm{x}: \mathrm{T}$, claiming the existence of a value $\mathrm{x}$ of type $\mathrm{T}$ in any module implementing this signature. Depending on $\mathrm{T}$, it corresponds either to an OCAML abstract type or to an OCAML value. A module or a functor body may contain logical properties and proofs. This is of course a novelty when compared to OCAML.

\subsection{Extraction}

The CoQ extraction mechanism [1213 handles naturally the module system: COQ modules are extracted to OCAML modules, COQ signatures to OCAML signatures and CoQ functors to OCAML functors.

The first task of the extraction is to introduce a distinction between terms and types, since CoQ has a unified notion of terms. For instance, a Parameter declaration may either be extracted to a type declaration or to a value declaration, depending on its type.

Then the extraction removes all the logical parts that "decorate" the computationally relevant terms: logical justifications, preconditions, postconditions, etc. These logical parts are not needed for the actual computation. The detection of these logical parts is done accordingly to the CoQ sorts. A fundamental principle of COQ is that any CoQ term belongs either to the logical sort Prop, or to the informative sorts Set and Type. The extraction follows this duality to decide which (sub-)terms must be erased.

\section{Specifying a Finite Sets Library}

\subsection{Ordered Types}

Similarly to OCAML code, our implementations are parameterized by the type of elements and its total ordering function. The OCAML ordering functions return integers for efficiency reasons. The COQ ordering functions could simply return into a three values enumeration type, such as

Inductive Compare: Set := Lt: Compare | Eq: Compare | Gt: Compare.

However, it is more idiomatic to introduce two predicates lt and eq- $1 \mathrm{t}$ for the strict order relation and eq for the equality - and to have constructors for 


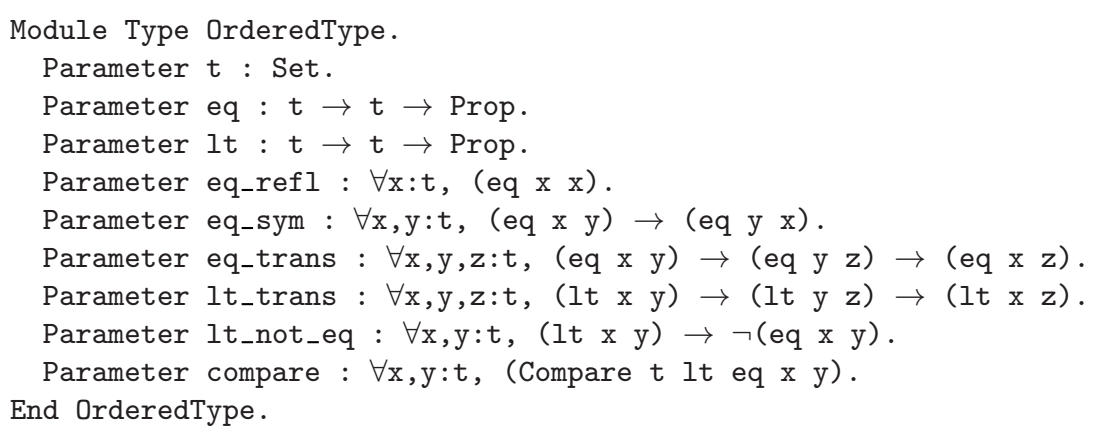

Fig. 2. COQ signature for ordered types

the inductive type Compare carrying proofs of the corresponding relations. Since this type is going to be reused at several places, we make it polymorphic with respect to the type $\mathrm{X}$ of elements and to the relations $1 \mathrm{t}$ and eq:

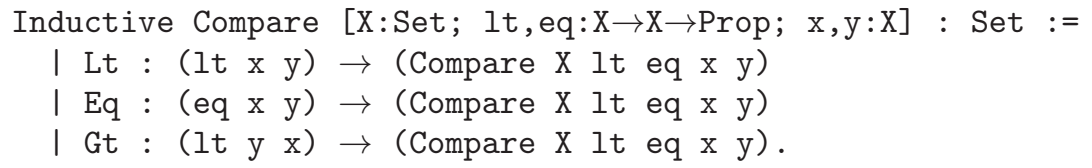

Note that this type is in sort Set while lt and eq are in sort Prop. Thus the informative contents of Compare is a three constant values type, with the same meaning as integers in OCAML comparison functions.

Then we introduce a signature OrderedType for ordered types. First, it contains a type $t$ for the elements, which is clearly informative and thus in sort Set. Then it equips the type $t$ with an equality eq and a strict order relation lt, together with a decidability function compare such that (compare $\mathrm{x} y$ ) has type (Compare $t$ lt eq $\mathrm{x} y$ ).

Finally, it contains a minimal set of logical properties expressing that eq is an equivalence relation and that $1 t$ is a strict order relation compatible with eq. The final signature OrderedType is given Figure 2, Note that compare provides the totality of the order relation.

Many additional properties can be derived from this set of axioms, such as the antisymmetry of 1t. Such properties are clearly useful for the forthcoming proof developments. Instead of polluting the OrderedType signature with all of them, we build a functor that derives these properties from OrderedType:

Module OrderedTypeFacts [0:OrderedType].

Lemma 1t_not_gt : $\forall \mathrm{x}, \mathrm{y}: 0 . t,(0.1 t \mathrm{x} y) \rightarrow \neg(0.1 t \mathrm{y} x)$. ...

End OrderedTypeFacts.

This way, the user only has to implement a minimal signature and all the remaining is automatically derived. 


\subsection{Finite Sets}

Reproducing the OCAmL Set.S signature in CoQ is not immediate. First of all, four functions are not applicative. One is iter, which iterates a side-effects-only function over all elements of a set; we simply discard this function. The others are min_elt, max_elt and choose, which respectively return the minimum, the maximum and an arbitrary element of a given set, and raise an exception when the set is empty; we slightly change their type so that they now return into a sum type.

Then we face the problem of specifications: they are given as comments in the Ocaml files, and are more or less precise. Sometimes the behavior is even left partly unspecified, as for fold. The remaining of this section explains how these informal specifications are translated into CoQ.

Finally, we face a question of style. There are indeed several ways of defining, specifying and proving correct a function in CoQ. Basically, this can be done in two steps - we first define a purely informative function and then we prove it correct - or in a single one - we use dependent types to have the function returning its result together with the proof that it is correct. Our formalization actually provides both styles, with bridge functors to go from one to the other, allowing the user to choose what he or she considers as the most convenient.

Non-dependent signature. We first introduce a signature S containing purely informative functions together with axioms. It is very close to the OCAML signature. It introduces an ordered type for the elements:

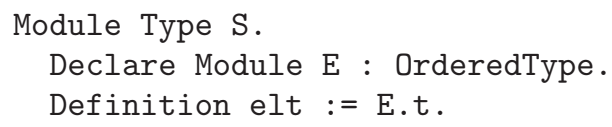

Then it introduces an abstract type $\mathrm{t}$ for sets:

Parameter $t$ : Set.

All operations have exactly the same types as in OCAML (see Figure 1), apart from min_elt, max_elt and choose:

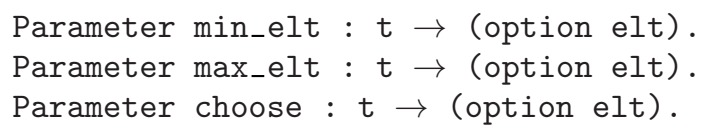

and compare which uses the Compare type introduced in Section 3.1 and refers to two predicates eq and $1 t$ also declared in the interface:

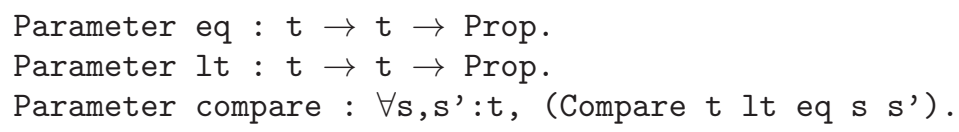

The five properties of eq and It are declared, making this interface a subtype of OrderedType and thus allowing a bootstrap to get sets of sets. To specify all the operations, a membership predicate is introduced: 
Parameter In : elt $\rightarrow$ t $\rightarrow$ Prop.

We could have used the mem operation for this purpose, using (mem $x \mathrm{~s}$ )=true instead of ( $\ln \mathrm{x} \mathrm{s}$ ), but it is more idiomatic in COQ to use propositions rather than boolean function:2. Moreover, it gives the implementor the opportunity to define a membership predicate without referring to mem, which may ease the correctness proof. An obvious property of In with respect to the equality E.eq is declared:

Parameter eq_In : $\forall \mathrm{s}: t, \forall \mathrm{x}, \mathrm{y}:$ elt, $($ E.eq $\mathrm{x} y) \rightarrow($ In $\mathrm{x} s) \rightarrow($ In $\mathrm{y} s)$.

Operations are then axiomatized using the membership predicate. The value empty and the operations mem, is_empty, add, remove, singleton, union, inter, diff, equal, subset, elements, min_elt, max_elt and choose have obvious specifications. The remaining six operations, namely filter, cardinal, fold, for_all, exists and partition, are not so simple to specify and deserve a bit of explanation.

Filtering and test functions. In the OCAML standard library, the filter operation is specified as follows:

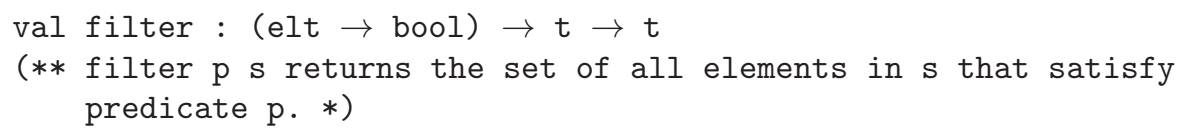

This is slightly incorrect, as the predicate $\mathrm{p}$ could return different values for elements identified by the equality E. eq over type elt. The predicate $\mathrm{p}$ has to be compatible with E.eq in the following way:

$$
\begin{gathered}
\text { Definition compat_bool }[\mathrm{p}: \mathrm{elt} \rightarrow \mathrm{bool}]:= \\
\forall \mathrm{x}, \mathrm{y}: \mathrm{elt}, \quad(\text { E.eq } \mathrm{x} y) \rightarrow(\mathrm{p} \mathrm{x})=(\mathrm{p} \mathrm{y}) .
\end{gathered}
$$

Then filter can be formally specified with the following three axioms:

$$
\begin{aligned}
& \text { Parameter filter_1 : } \forall \mathrm{s}: \mathrm{t}, \forall \mathrm{x}: \mathrm{elt}, \forall \mathrm{p}: \mathrm{elt} \rightarrow \mathrm{bool} \text {, } \\
& (\text { compat_bool p) } \rightarrow(\text { In } x \text { (filter } \mathrm{p} \mathrm{s})) \rightarrow(\text { In } \mathrm{x} s) \text {. } \\
& \text { Parameter filter_2 : } \forall \mathrm{s}: t, \forall \mathrm{x}: \text { elt, } \forall \mathrm{p}: \mathrm{elt} \rightarrow \mathrm{bool} \text {, } \\
& (\text { compat_bool p) } \rightarrow(\text { In } x \text { (filter } p s)) \rightarrow(p x)=\text { true. } \\
& \text { Parameter filter_3 : } \forall \mathrm{s}: \mathrm{t}, \forall \mathrm{x}: \mathrm{elt}, \forall \mathrm{p}: \mathrm{elt} \rightarrow \mathrm{bool} \text {, } \\
& (\text { compat_bool } \mathrm{p}) \rightarrow(\operatorname{In} \mathrm{x} s) \rightarrow(\mathrm{p} x)=\operatorname{true} \rightarrow(\operatorname{In} \mathrm{x} \text { (filter } \mathrm{p} \mathrm{s})) \text {. }
\end{aligned}
$$

Note that it leaves the behavior of filter unspecified whenever $\mathrm{p}$ is not compatible with E.eq. Operations for_all, exists and partition are specified in a similar way.

Folding and cardinal. Specifying the fold operation poses another challenge. The OCAML specification reads:

$$
\begin{aligned}
& \text { val fold : (elt } \rightarrow \text { 'a } \rightarrow \text { 'a) } \rightarrow \text { t } \rightarrow \text { 'a } \rightarrow \text { 'a } \\
& \text { (** fold } f \mathrm{~s} \text { a computes }(f \mathrm{xN} \ldots \text { ( } f \mathrm{x} 2(\mathrm{f} \mathrm{x} 1 \mathrm{a})) \ldots \text { ), where } \\
& \mathrm{x} 1 \ldots \mathrm{xN} \text { are the elements of } \mathrm{s} \text {. The order in which elements } \\
& \text { of } s \text { are presented to } f \text { is unspecified. *) }
\end{aligned}
$$

${ }^{2}$ Unlike most HOL-based systems, CoQ does not identify propositions and booleans. 
To resemble the OCAML specification as much as possible, we declare the existence of a list of elements without duplicate - the list $\mathrm{x} 1, \ldots, \mathrm{xN}$ above - and we reuse the existing folding operation fold_right over lists:

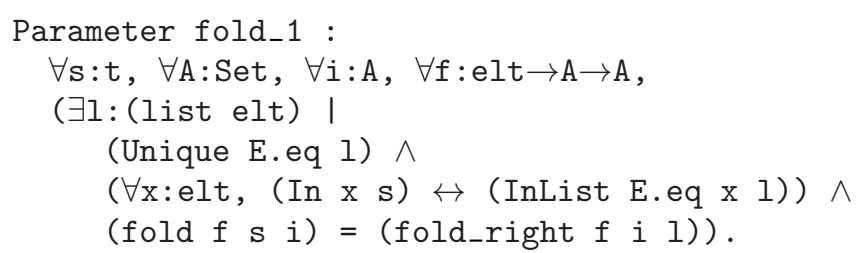

Unique is a predicate expressing the uniqueness of elements within a list with respect to a given equality, here $\mathrm{E}$. eq. The cardinal operation is specified in a similar way, using the operation length over lists. Note that cardinal could be defined with fold; this will be discussed later in Section 3.3 .

Dependent signature. We introduce a second signature for finite sets, Sdep. It makes use of dependent types to mix computational and logical contents, in a CoQ idiomatic way of doing. The part of the signature related to the type $t$ and the relations eq, It and In is exactly the same as for signature S. Then each operation is introduced and specified with a single declaration. For instance, the empty set is declared as follows:

Parameter empty : $\{$ s:t | $\forall$ a:elt, $\neg($ In a s) $\}$.

which must read "there exists a set s such that ...". Similarly, the operation add is declared as:

Parameter add : $\forall \mathrm{x}:$ elt, $\forall \mathrm{s}: \mathrm{t}$,

$$
\left\{\mathrm{s}^{\prime}: \mathrm{t} \mid \forall \mathrm{y}: \text { elt, }\left(\text { In } \mathrm{y} \mathrm{s}^{\prime}\right) \leftrightarrow((\text { E.eq } \mathrm{y} \mathrm{x}) \vee(\text { In } \mathrm{y} \mathrm{s}))\right\} .
$$

and so on for all other operations.

Bridge functors. Signatures S and Sdep can be proved equivalent in a constructive way. Indeed, we can implement two bridge functors between the two signatures. The first one is implementing the signature Sdep given a module implementing the signature $\mathrm{S}$ :

Module DepOfNodep [M:S] <: Sdep with Module E := M.E.

...

End DepOfNodep.

and the second one is implementing the signature $\mathrm{S}$ given a module implementing the signature Sdep:

Module NodepOfDep [M:Sdep] <: S with Module E := M.E. ...

End NodepOfDep.

The practical interest is obvious: the user may prefer one style of programming/proving with COQ while a particular implementation of finite sets is available with the other style. Applying the appropriate functor provides the desired interface. 


\subsection{Additional Properties}

Signatures S and Sdep intend to be minimal. Many additional properties can be derived. They may involve the set operations separetely or together, as in the following fact:

cardinal (union a b) + cardinal (inter $a b)=$ cardinal $a+$ cardinal $b$

Similarly to what we did for OrderedType in Section 3.1, we gather all such properties in a functor taking a module of signature $\mathrm{S}$ as argument:

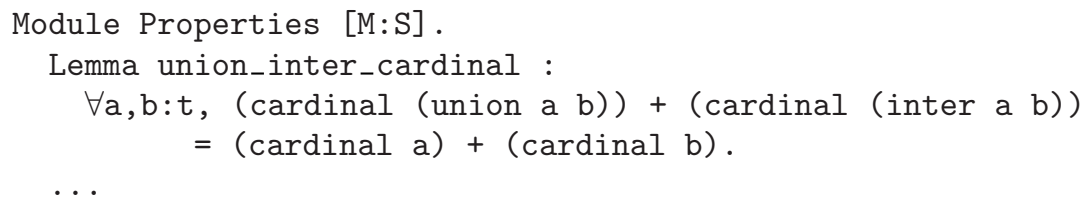

End Properties.

\section{Verifying Finite Sets Implementations}

Implementing and verifying a set library with all operations introduced so far is not necessarily difficult: indeed, all operations can be coded using the four primitive operations empty, add, remove and fold. However, most operations can be coded more efficiently in a direct way, at the extra cost of a more difficult formal proof.

In this section, we present the formal verification of three different implementations using respectively sorted lists, AVL trees and red-black trees. These three implementations are functors taking an ordered type $\mathrm{X}$ as argument.

\subsection{Sorted Lists}

Sets implemented as sorted lists offer poor performances. However, there are at least two reasons to start with such an implementation. First, this is a quick way to debug our signature $\mathrm{S}$ and, when done, to show its logical consistency-we indeed rephrased several specifications while doing this first verification. Second, some of the operations over lists are reused later in the code or verification of the other two implementations based on binary trees. The verification is (almost) straightforward.

\subsection{AVL Trees}

The next implementation to be verified is the Set module from OcAML standard library 2]. This is a heavily used library, including in OCAML's own code. It implements sets using AVL trees [4], that are binary search trees where the difference between the heights of any two sibling trees can not exceed a given value $\Delta$. Although $\Delta=1$ is an admissible choice [4, the OCAML implementation relaxes it to $\Delta=2$, making a compromise between the overall balancing and the cost of rebalancing when inserting or deleting. 
The CoQ formalization implements signature Sdep, following OcAML code as close as possible. First a type for trees is introduced:

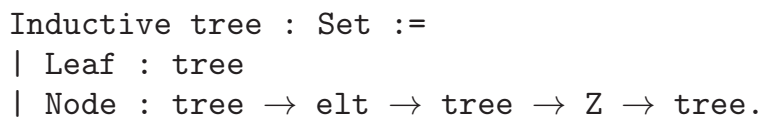

The height is stored for greater efficiency, using the CoQ type $\mathrm{Z}$ for arbitrary precision integers. Then we introduce two inductive predicates

$$
\begin{aligned}
& \text { Inductive bst : tree } \rightarrow \text { Prop }:=\ldots \\
& \text { Inductive avl : tree } \rightarrow \text { Prop }:=\ldots
\end{aligned}
$$

where bst (resp. avl) is the property of being a binary search tree (resp. a balanced tree). Finally, the type $t$ for sets is a record containing a tree and proofs that it is a balanced binary search tree:

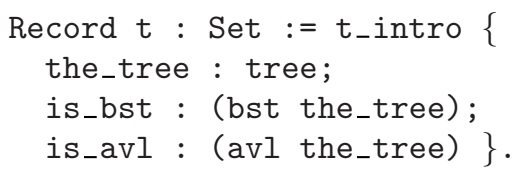

Properties bst and avl could have been defined simultaneously but separating them eases the proofs since most of the time one of the two is not relevant for the property to be proved.

Verification. Verifying the operations is mostly a matter of finding the precise specifications, where the OCAML code is only providing a few laconic comments. For instance, one of the internal function (bal $1 \times x$ ) is informally specified as

"Same as create, but performs one step of rebalancing if necessary. Assumes 1 and $r$ balanced."

but its precise specification is (among other things):

"Assumes $\mid$ (height 1$)-$ (height $r) \mid \leq 3$. The size of the returned tree is either $\max ($ height 1 , height $r$ ) or $\max ($ height 1 , height $r)+1$, and is always the latter when $\mid$ (height 1 ) - (height $r$ ) $\mid \leq 2$.

Looking for these specifications, we actually discovered balancing bugs in OCAML code: two internal functions were building incorrectly balanced trees while they were supposed to. (The sets which were built were correct, though, i.e. were containing the right elements.) Patches have been quickly provided by the OCAML team and we could verify the new code without trouble.

A termination challenge. Only one operation poses a real verification challenge: the compare function providing a total ordering over sets. The idea is quite simple. Comparing two sets is just a matter of comparing the sorted lists of their elements in a lexicographic way. But the algorithm used is tricky. Instead of first building the two lists, the code is building them lazily, as soon as elements are needed for the comparison, using a technique of deforestation 15. The problem is generalized to the comparison of two lists of trees, done as follows: 


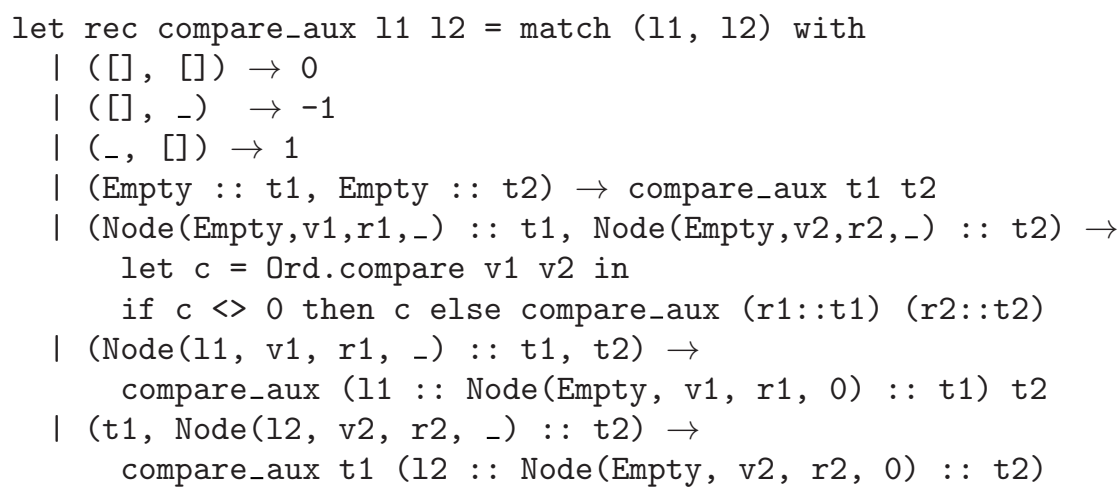

Proving the termination of this function is hard. Indeed, the last two cases may recursively call compare_aux on "bigger" arguments when 11 (resp. 12) is Empty. The reason why it terminates involves a global argument: the first elements of the lists will eventually become both Empty and will then fall into the fourth case of the pattern matching. Fortunately, the code can be slightly changed to recover a simpler termination argument, at the extra cost of two additional cases but without any loss of efficiency. This modified version is proved correct.

\subsection{Red-Black Trees}

Red-black trees [8] are another kind of balanced binary search trees. Nodes are colored either red or black and any red-black tree must satisfy the following two invariants:

- A red node has no red child;

- Every path from the root to a leaf contains the same number of black nodes.

Okasaki nicely introduces red-black trees in a functional setting [14 but only the membership and insertion operations are given. $\mathrm{Xi}$ specifies this code in DEPENDENT ML 16] but it is also restricted to the insertion operation. Even Adams general approach to balanced binary search trees [3] does not apply nicely to red-black trees. Generally speaking, we could not find a comprehensive implementation of finite sets using red-black trees and we wrote our own. This code is available from the web site of the formalization.

As for AVL trees, the COQ formalization implements signature Sdep following OCAML code as close as possible. First, colored trees are defined:

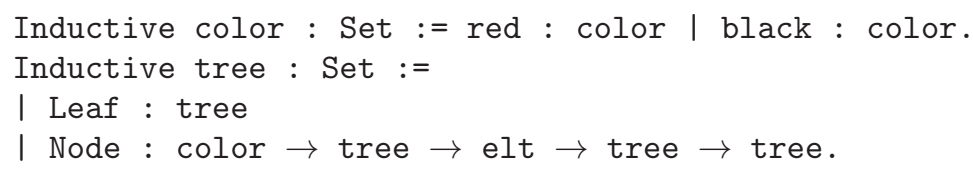

The binary search tree property bst is similar to the one for AVL trees. Then the red-black trees invariant is introduced as an inductive predicate rbtree parameterized by the height of black nodes. Finally, everything is collected into a record type: 


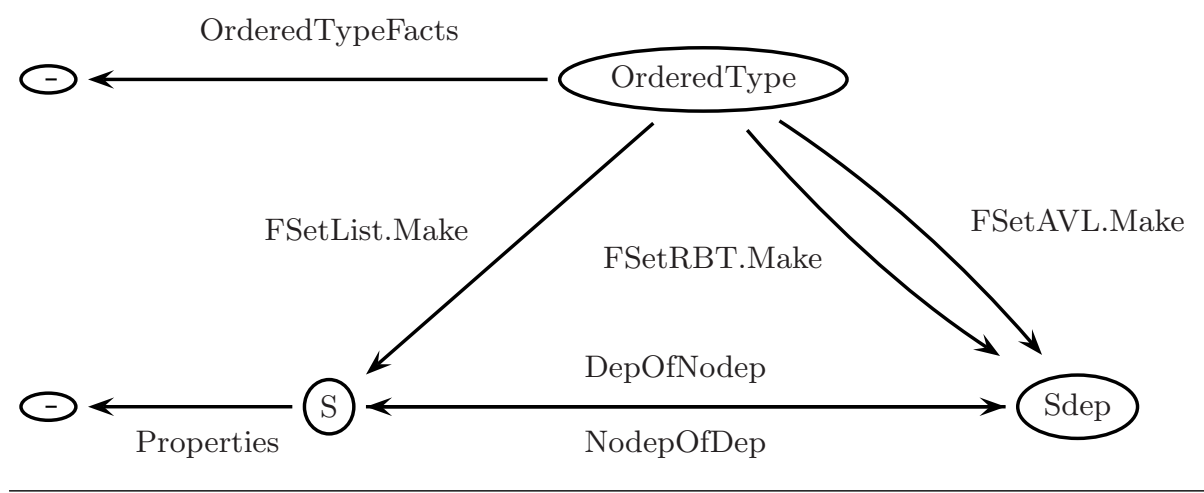

Fig. 3. The five signatures and the seven functors

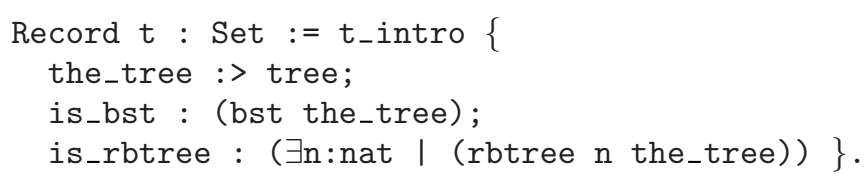

Again, the formalization is roughly a matter of finding the right specification for each function, followed by a quite long process of COQ tactics scripting (figures are given in the conclusion). Some proofs from the AVL trees formalization could be reused with slight modifications (e.g. the compare operation).

\section{Conclusion}

In this article we presented the full formalization in COQ of three applicative implementations of finite sets libraries, including AVL and red-black trees. To our knowledge this is the first formal proof of a full set of operations over these two kinds of balanced binary search trees. This is also the first formal proof of a functorized piece of code.

This article also demonstrates the benefits of modules and functors in a logical framework, and their relevance for program proving. More precisely, the adequacy between the OCAML and COQ module systems allows the formalization of significant pieces of code. Correct-by-construction functorized code can be obtained using CoQ extraction, which is a real improvement.

Overall picture. Figure 3 summarizes the dependencies between the five signatures and the seven functors used in this formalization. The following table details the size of the formalization, in terms of the size of code proved correct and size of the CoQ development. The formalization roughly amounts to one man-month. 


\begin{tabular}{|l|r|r|r|r|r|}
\hline & specs & lists & AVL & RBT & total \\
\hline lines of code & & 114 & 231 & 314 & 545 \\
\hline lines of Coq specs & 1160 & 375 & 532 & 405 & 2472 \\
\hline lines of Coq proofs & 1900 & 537 & 1800 & 1280 & 5517 \\
\hline
\end{tabular}

Extraction and benchmark. Once the formalization done, OCAML code can be automatically extracted from the proofs 1213. Thus it can be compared to the original code. We run a little benchmark comparing OCAML's set module (AVL), the extraction of its formalization ( $\varepsilon$-AVL), a manual implementation of red-black trees (RBT) and the extraction of its formalization $(\varepsilon-\mathrm{RBT})$. The benchmark consists in testing operations on randomly generated sets of various sizes. Timings are shown below (in seconds).

\begin{tabular}{|l|r|r|r|r|r|r|r|r|r|r|r|}
\hline & add-1 & add-2 & mem-1 & mem-2 & rem-1 & rem-2 & union & inter & diff & subset & cmp \\
\hline AVL & 3.01 & 15.50 & 9.52 & 11.10 & 4.67 & 4.20 & 11.40 & 4.76 & 4.68 & 11.50 & 11.50 \\
\hline$\varepsilon$-AVL & 13.80 & 53.20 & 10.20 & 11.70 & 18.70 & 17.70 & 46.50 & 17.70 & 17.70 & 46.30 & 45.70 \\
\hline RBT & 3.38 & 13.40 & 11.40 & 13.00 & 5.92 & 4.81 & 12.10 & 4.76 & 4.71 & 11.20 & 11.70 \\
\hline$\varepsilon-$ RBT & 4.02 & 16.70 & 12.10 & 13.60 & 6.85 & 5.58 & 17.30 & 6.19 & 5.76 & 14.00 & 13.90 \\
\hline
\end{tabular}

The timings are very close, apart from $\varepsilon$-AVL when trees are built, that is for all operations except mem. The reason is that arithmetical computations over heights are done using COQ arbitrary precision arithmetic extracted to OCAML, which can not compete with the hardware arithmetic used in OcAmL Set. We could parameterize the whole formalization of AVL trees with respect to the arithmetic used for computing heights, using yet another functor. But we would loose the benefits of the Omega tactic (the decision procedure for Presburger arithmetic) which is of heavy use in this development. A workaround would be an automatic substitution of a more realistic arithmetic for CoQ arithmetic at extraction time, e.g. hardware or arbitrary precision provided it has been verified. But this is not yet a COQ feature.

Acknowledgements. We are grateful to Xavier Leroy for suggesting the verification of OCAML's AVL trees and for having provided patches almost immediately. We also thank Benjamin Monate for the very nice user-interface CoQIDE and Diego Olivier Fernandez Pons for comments on implementing red-black trees.

\section{References}

1. The Coq Proof Assistant. http://coq.inria.fr/

2. The Objective Caml language. http://caml.inria.fr/

3. Stephen Adams. Functional pearls: Efficient sets - a balancing act. Journal of Functional Programming, 3(4):553-561, October 1993. Expanded version available as Technical Report CSTR 92-10, University of Southampton.

3 The benchmark sources can be obtained from the authors. 
4. G. M. Adel'son-Vel'skil and E. M. Landis. An algorithm for the organization of information. Soviet Mathematics-Doklady, 3(5):1259-1263, September 1962.

5. Jacek Chrząszcz. Implementing modules in the system Coq. In 16th International Conference on Theorem Proving in Higher Order Logics, University of Rome III, September 2003.

6. Jacek Chrząszcz. Modules in Type Theory with generative definitions. PhD thesis, Warsaw University and Université Paris-Sud, 2003. To be defended.

7. Judicaël Courant. A Module Calculus for Pure Type Systems. In Typed Lambda Calculi and Applications 97, Lecture Notes in Computer Science, pages 112 - 128. Springer-Verlag, 1997.

8. Leo J. Guibas and Robert Sedgewick. A dichromatic framework for balanced trees. In 19th Annual Symposium on Foundations of Computer Science, pages 8-21, Ann Arbor, Michigan, 16-18 October 1978. IEEE.

9. Robert Harper and Mark Lillibridge. A type-theoretic approach to higher-order modules with sharing. In Conference record of POPL '94: 21st ACM SIGPLANSIGACT Symposium on Principles of Programming Languages, pages 123-137, Portland, OR, January 1994.

10. Ralf Hinze. Constructing red-black trees. In editor Chris Okasaki, editor, Proceedings of the Workshop on Algorithmic Aspects of Advanced Programming Languages, WAAAPL'99, pages 89-99, Paris, France, September 1999. Also technical report of Columbia University, CUCS-023-99.

11. Xavier Leroy. A modular module system. Journal of Functional Programming, 10(3):269-303, 2000.

12. Pierre Letouzey. A New Extraction for Coq. In Herman Geuvers and Freek Wiedijk, editors, Types for Proofs and Programs, Second International Workshop, TYPES 2002, Berg en Dal, The Netherlands, April 24-28, 2002, volume 2646 of Lecture Notes in Computer Science. Springer-Verlag, 2003.

13. Pierre Letouzey. Programmation fonctionnelle certifiée en Coq. PhD thesis, Université Paris Sud, 2003. To be defended.

14. Chris Okasaki. Purely Functional Data Structures. Cambridge University Press, 1998.

15. Philip Wadler. Deforestation: transforming programs to eliminate trees. Theoretical Computer Science, 73:231-248, 1990.

16. Hongwei Xi. Dependently Typed Data Structures. In Proceedings of Workshop of Algorithmic Aspects of Advanced Programming Languages (WAAAPL '99), pages 17-32, Paris, September 1999. 